COLO-HEP-360

\title{
Spin Glasses on Thin Graphs
}

\author{
C.F. Baillie \\ Dept of Computer Science, University of Colorado \\ Boulder, CO 80309, USA
}

W. Janke

Institut fur Physik

Johannes Gutenberg Universitat

D 55099 Mainz, Germany

\author{
D.A. Johnston and P. Plecháč \\ Dept. of Mathematics \\ Heriot-Watt University \\ Riccarton \\ Edinburgh, EH14 4AS, Scotland
}

9 May 1995

\begin{abstract}
In a recent paper 11 we found strong evidence from simulations that the Ising antiferromagnet on "thin" random graphs - Feynman diagrams - displayed a mean-field spin glass transition. The intrinsic interest of considering such random graphs is that they give mean field theory results without long range interactions or the drawbacks, arising from boundary problems, of the Bethe lattice. In this paper we reprise the saddle point calculations for the Ising and Potts ferromagnet, antiferromagnet and spin glass on Feynman diagrams. We use standard results from bifurcation theory that enable us to treat an arbitrary number of replicas and any quenched bond distribution. We note the agreement between the ferromagnetic and spin glass transition temperatures thus calculated and those derived by analogy with the Bethe lattice or in previous replica calculations.

We then investigate numerically spin glasses with a $\pm J$ bond distribution for the Ising and $Q=3,4,10,50$ state Potts models, paying particular attention to the independence of the spin glass transition from the fraction of positive and negative bonds in the Ising case and the qualitative form of the overlap distribution $P(q)$ for all of the models. The parallels with infinite range spin glass models in both the analytical calculations and simulations are pointed out.

Submitted to Nucl. Phys. B. [FS]
\end{abstract}




\section{Introduction and Analytical Calculations}

The analytical investigation of spin glasses on random graphs of various sorts has a long and honourable history [2, 3], though there has been little in the way of numerical simulations 1. Random graphs with a fixed or fixed average connectivity have a locally tree like structure, which means that loops in the graph are predominantly large, so Bethe-lattice-like [5] (ie mean field) critical behaviour is expected for spin models on such lattices. Given this, the analytical solution for a spin model or, in particular, a spin glass on a Bethe lattice [6, 7] can be translated across to the appropriate fixed connectivity random lattice. Alternatively, a replica calculation can be carried out directly in some cases for spin glasses on various sorts of random lattices. For the case of Ising spins on a fixed connectivity random lattice we arrive at the following prediction for the ferromagnetic transition temperature, if one exists

$$
\int_{-\infty}^{\infty} P(J) \tanh \left(\beta_{F M} J\right) d J=\frac{1}{z-1}
$$

where $P(J)$ is the quenched probability distribution of bonds in the model, $z$ is the connectivity and $\beta_{F M}$ is the (inverse) transition temperature. A similar relation is predicted for the spin glass transition temperature $\beta_{S G}$

$$
\int_{-\infty}^{\infty} P(J) \tanh ^{2}\left(\beta_{S G} J\right) d J=\frac{1}{z-1} .
$$

From equs.(1ㄴ:2) we would expect to find a ferromagnetic transition when $P(J)=p \delta(J-1)+(1-p) \delta(J+1)$ at

$$
(2 p-1) \tanh \left(\beta_{F M}\right)=\frac{1}{(z-1)}
$$

or a spin glass transition at

$$
\tanh ^{2}\left(\beta_{S G}\right)=\frac{1}{(z-1)} .
$$

depending on which critical temperature was lower. The global order parameter that is necessary to describe the system in the spin glass case [2, 3] appears as the fourier transform of the local field distribution.

A rather different way of looking at the problem of spin models on random graphs was put forward in [9], where it was observed that the requisite ensemble of random graphs could be generated by considering the Feynman diagram expansion for the partition function of the model. For an Ising ferromagnet with Hamiltonian

$$
H=\beta \sum_{<i j>} \sigma_{i} \sigma_{j}
$$

where the sum is over nearest neighbours on three-regular random graphs (ie $\phi^{3}$ Feynman diagrams), the partition function is given by

$$
Z_{n}(\beta) \times N_{n}=\frac{1}{2 \pi i} \oint \frac{d \lambda}{\lambda^{2 n+1}} \int \frac{d \phi_{+} d \phi_{-}}{2 \pi \sqrt{\operatorname{det} K}} \exp (-S)
$$

where $N_{n}$ is the number of undecorated graphs with $2 n$ points,

$$
N_{n}=\left(\frac{1}{6}\right)^{2 n} \frac{(6 n-1) ! !}{(2 n) ! !}
$$

$K$ is defined by

$$
K_{a b}=\left(\begin{array}{cc}
\sqrt{g} & \frac{1}{\sqrt{g}} \\
\frac{1}{\sqrt{g}} & \sqrt{g}
\end{array}\right)
$$

\footnotetext{
${ }^{1}$ For some recent simulations, see 4 .
} 
and the action itself is

$$
S=\frac{1}{2} \sum_{a, b} \phi_{a} K_{a b}^{-1} \phi_{b}-\frac{1}{3}\left(\phi_{+}^{3}+\phi_{-}^{3}\right) .
$$

where the sum runs over \pm indices. The coupling in the above is $g=\exp (2 \beta J)$ where $J=1$ for the ferromagnet and the $\phi_{+}$field can be thought of as representing "up" spins with the $\phi_{-}$field representing "down" spins. An ensemble of $z$-regular random graphs would simply require replacing the $\phi^{3}$ terms with $\phi^{z}$ and a fixed average connectivity could also be implemented with the appropriate choice of potential.

This approach was inspired by the considerable amount of work that has been done in recent years on $N \times N$ matrix 7 versions of such integrals which generate "fat" or ribbon graphs graphs with sufficient structure to carry out a topological expansion 10 because of the matrix index structure. The natural interpretation of such fat graphs as the duals of triangulations, quadrangulations etc. of surfaces has led to much interesting work in string theory and particle physics 111. The partition function here is a poor, "thin" (no indices, so no ribbons), scalar cousin of these, lacking the structure to give a surface interpretation to the graph. Such scalar integrals have been used in the past to extract the large $n$ behaviour of various field theories [12] again essentially as a means of generating the appropriate Feynman diagrams, so a lot is known about handling their quirks.

For the Ising ferromagnet on three-regular $\left(\phi^{3}\right)$ graphs, solving the saddle point equations at large $n$ shows that the critical behaviour appears as an exchange of dominant saddle point solutions to the saddle point equations

$$
\begin{aligned}
& \phi_{+}=\sqrt{g} \phi_{+}^{2}+\frac{1}{\sqrt{g}} \phi_{-}^{2} \\
& \phi_{-}=\sqrt{g} \phi_{-}^{2}+\frac{1}{\sqrt{g}} \phi_{+}^{2}
\end{aligned}
$$

at $g=\exp \left(2 \beta_{F M}\right)=3$. The high and low temperature solutions respectively are

$$
\begin{aligned}
\phi_{+}, \phi_{-} & =\frac{\sqrt{g}}{g+1} \\
\phi_{+}, \phi_{-} & =\frac{\sqrt{g}}{2(g-1)}\left(1 \pm \sqrt{\frac{g-3}{g+1}}\right)
\end{aligned}
$$

which give a low temperature magnetized phase that can be detected by a non-zero magnetization order parameter, which is defined in terms of the fields $\phi_{+}, \phi_{-}$as

$$
M=\frac{\phi_{+}^{3}-\phi_{-}^{3}}{\phi_{+}^{3}+\phi_{-}^{3}}
$$

The critical exponents for the transition can also be calculated in this formalism and, as expected, are mean field. In general a mean field transition appears at

$$
\exp \left(2 \beta_{F M}\right)=z /(z-2)
$$

on $\phi^{z}$ graphs, which is exactly the value predicted by equ(3) in the standard approaches.

To consider a non-trivial bond distribution it suffices to make the substitution [9]

$$
K_{a b} \rightarrow \int_{-\infty}^{\infty} K_{a b} P(J) d J
$$

in the saddle point equations. If we take the distribution $P(J)=p \delta(J-1)+(1-p) \delta(J+1)$, this has the effect of replacing $g$ by

$$
\frac{p g+1-p}{p+(1-p) g}
$$

\footnotetext{
${ }^{2} N$, the size of the matrix is not to be confused with $n$, the number of vertices in the graph!
} 
On $\phi^{z}$ graphs we find that this change in the coupling shifts the ferromagnetic transition point to

$$
\exp \left(2 \beta_{F M}\right)=\frac{(2 p-1)(z-1)+1}{(2 p-1)(z-1)-1}
$$

which is again identical to the value found from equ.(3).

This is rather surprising as a direct substitution of the weighted propagator coming from equ.(14) into the saddle point equations might be expected to correspond an annealed distribution of bonds because we are calculating with a finite number (one) of replicas. The calculations leading to equ.(3) and equ.(位), however, have taken quenched bond distributions by calculating with $k$ replicas and then taking the limit $k \rightarrow 0$.

\section{The Saddle Point Equations for Spin Glasses}

There are various possibilities for addressing spin glass order in the Feynman diagram approach. In 9] the entropy per spin was calculated for the Ising anti-ferromagnet on $\phi^{3}$ graphs and it was found to become negative for sufficiently negative $\beta$, which is often indicative of a spin glass transition. Similarly it was found that the factorized solution (which exists in the ferromagnetic and anti-ferromagnetic cases) broke down for higher moments $Z^{k}$ of the partition function, which is again indicative of a spin glass transition. The temperature at which this happened appeared to be converging to a finite value as the moments increased, unlike the random energy model where a similar calculation gives a temperature that diverges as $\sqrt{k}[13]$.

We now look at the calculation of $Z^{2}$ in a little more detail. We can put two Ising models on each Feynman diagram by taking four fields $\phi_{++}, \phi_{+-}, \phi_{-+}, \phi_{--}$, where the double subscript now covers both replicas, and enlarging the inverse propagator to

$$
K_{a b}=\left(\begin{array}{cccc}
g & 1 & 1 & \frac{1}{g} \\
1 & g & \frac{1}{g} & 1 \\
1 & \frac{1}{g} & g & 1 \\
\frac{1}{g} & 1 & 1 & g
\end{array}\right)
$$

If we solve explicitly the saddle point equations for the two replica system with $P(J)=p \delta(J-1)+$ $(1-p) \delta(J+1)$ we find a $p$-independent solution appearing at $g=5.8284 .$. and a mirror solution at the corresponding antiferromagnetic value given by the inverse of this, $g=0.1716 \ldots$. The second solution, $g=0.1716 \ldots$, was also observed in [9] as the point where the factorized solution broke down for the antiferromagnet with $k=2$. Remarkably, as we are looking at a finite number (two) of replicas here, $g=5.8284, g=0.1716$ are precisely the values given by eqn.(武) for $\beta_{S G}$, where the limit $k \rightarrow 0$ has been taken to obtain the spin glass transition temperature. Thus, just as for the ferromagnetic transition, the quenched $k \rightarrow 0$ results are appearing already at $k \neq 0$. Using numerical routines to investigate the structure of the saddle point equations for more Ising replicas reveals that the solutions at $\beta_{F M}$ and $\beta_{S G}$ still appear at higher $k$ as bifurcations from the symmetric high temperature solution.

This is not quite the whole story. As already noted in [9] for $k=3,4 \ldots$ we hit a different sort of critical behaviour which takes us off the symmetric solution branch to a replica symmetric solution before reaching $\beta_{S G}$. At these points a first order (jump in $q$ ) transition occurs. This behaviour is identical to that of the Ising replica magnet [14], which is effectively the SK model at a finite number of replicas for a suitable choice of parameters. In this the $k=2$ transition is second order and the transition temperature coincides with the $k=0$ value, whereas the $k \geq 3$ transitions are first order and occur at higher temperatures.

We can attempt to analyse the saddle point equations for any number $k$ of Ising replicas in the case of purely ferromagnetic or antiferromagnetic couplings using bifurcation theory methods because the tensor product structure of the propagator $K_{a b}$ is preserved in these cases 3 which facilitates the calculation.

\footnotetext{
${ }^{3}$ This is just a more formal statement of the observation in 9 that a solution given by the product of $k=1$ solutions existed in these cases.
} 
We will not, however, see first order transitions such as those discussed above because the bifurcation structure only tells us about continuous transitions from the symmetric high temperature solution.

If we denote the $2 \times 2$ propagator in equ. $(7)$ as $K(g)$ for brevity, the saddle point equations for the $k$ replica case may now be written schematically as

$$
\frac{\partial S_{k}}{\partial \vec{\phi}} \simeq \vec{\phi}-\otimes^{k} K(g) \vec{\phi}^{2}=0
$$

where $\vec{\phi}, \vec{\phi}^{2}$ are now $2^{k}$ dimensional vectors, the tensor product is taken over $k$ copies of $K$ and the action for $k$ replicas is denoted by $S_{k}$. We have abused the notation slightly in equ.(18) as the left hand side is really $\otimes^{k} K(g) \partial S_{k} / \partial \vec{\phi}$ because we have multiplied by $\otimes^{k} K(g)$ to get rid of the inverse factor coming from the quadratic term in $S_{k}$, which simplifies the analysis of the saddle point equations. We will continue the abuse in the discussion below.

For the antiferromagnetic case $g$ is simply replaced by $1 / g$ throughout. We restrict ourselves to investigating the symmetric branch (and hence ignore the first order solutions that appear for $k>2$ ). A symmetric, high temperature solution can be constructed by taking products of the $k=1$ symmetric solution in equ.(10) $(t, t)$ where

$$
t=\frac{\sqrt{g}}{g+1}
$$

Standard theory 15 then shows that a bifurcation from the symmetric high temperature solution (ie phase transition) is expected when the Hessian $\operatorname{det}\left(\partial^{2} S_{k} / \partial \vec{\phi}^{2}\right)$ is equal to zero. More explicitly

$$
\operatorname{det}\left(\frac{\partial^{2} S_{k}}{\partial \vec{\phi}^{2}}\right)=\operatorname{det}\left(2 t^{k} K_{k}-1\right)
$$

where we have denoted the tensor product of $k$ copies of $K$ by $K_{k}$. If we absorb the factors of $t$ into $K$ and denote $\tilde{K}=(\sqrt{g}+1 / \sqrt{g})^{-1} K$ we thus have

$$
\operatorname{det}\left(\frac{\partial^{2} S_{k}}{\partial \vec{\phi}^{2}}\right)=\operatorname{det}\left(2 \tilde{K}_{k}-1\right)=0
$$

as our bifurcation equation. The extension to $z$-regular $\left(\phi^{z}\right)$ graphs is trivial. For these the $k=1$ high temperature solution is $(\tilde{t}, \tilde{t})$ where

$$
\tilde{t}=\left(\frac{\sqrt{g}}{g+1}\right)^{1 /(z-2)}
$$

and the Hessian becomes

$$
\operatorname{det}\left(\frac{\partial^{2} S_{k}}{\partial \vec{\phi}^{2}}\right)=\operatorname{det}\left((z-1)\left(\tilde{t}^{z-2}\right)^{k} K_{k}-1\right)
$$

so the net effect is simply to replace 2 by $z-1$ in the bifurcation equation.

The matrix $\tilde{K}$ has two eigenvalues 1 and $(g-1) /(g+1)=\tanh (\beta)$. From the algebraic structure of $\tilde{K}_{k}$ we can readily calculate the eigenvalues $\lambda_{m}=\tanh (\beta)^{m}, m=0,1,2, \ldots k$ where each eigenvalue has multiplicity $\left(\begin{array}{l}k \\ m\end{array}\right)$ and express (taking $z=3$ )

$$
\operatorname{det}\left(\frac{\partial^{2} S_{k}}{\partial \vec{\phi}^{2}}\right)=\prod_{m=0}^{k}\left(2 \tanh (\beta)^{m}-1\right)^{\left(\begin{array}{c}
k \\
m
\end{array}\right)}=0 .
$$

The roots of the bifurcation equation are thus

$$
\tanh (\beta)=2^{-1 / m} m=1,2, \ldots k
$$

reproducing equ.(3) for $p=1$ and $z=3$. In terms of $\beta=(1 / 2) \log (g)$ we can write this as

$$
\beta_{m}=\frac{1}{2} \log \left(\frac{2^{1 / m}+1}{2^{1 / m}-1}\right) \quad m=1,2 \ldots k
$$


where we have retained the label $m$ on $\beta$. The above analysis holds for the ferromagnet where $g=$ $\exp (2 \beta) \geq 1$. In the case of a pure antiferromagnet $g=\exp (2 \tilde{\beta}) \leq 1$ and $\tanh (\beta)$ is negative, so we can only find roots of the bifurcation equation for $m$ even.

$$
\tilde{\beta}_{m}=\frac{1}{2} \log \left(\frac{2^{1 / m}-1}{2^{1 / m}+1}\right) \quad m=2,4 \ldots k
$$

We have thus recovered both $\beta_{F M}=\beta_{1}(g=3)$ for the ferromagnet and $\beta_{S G}=\tilde{\beta}_{2}(g=0.1716)$ for the antiferromagnet in this approach.

A generic feature of the above solutions is immediately obvious - once a bifurcation appears at some $k$ it is always present for a greater number of replicas. This suggests a possible resolution of the puzzle of why the quenched transition temperatures are appearing already at finite $k$ : The appropriate transition is encountered at the same temperature for all $k$, so the analytical continuation $k \rightarrow 0$ is trivial. In the spin glass case the first order transitions for $k \geq 3$ are the fly in the ointment, as these are encountered before the putative continuous transition at $\beta_{S G}$. Looking explicitly at the replica symmetric solution for $k=3[9]$ shows that the the replica symmetric banch appears in a first order transition at $g \simeq 0.19 \ldots$ (in the antiferromagnet) before the symmetric branch takes over again below this.

It is rather amusing that we have encountered a "c=1 (2 Ising model) barrier" in this context. Calculations are still possible here for $k>2$, but there is certainly a change of behaviour. In the case of spin models on annealed ensembles of fat graphs analytical calculations have struggled to get beyond $c=1$, whereas simulations have so far failed to see much difference between $c<1$ and $c>1$.

Although the tensor product structure of $K_{k}$ is lost when $p \neq 0,1$ and the saddle point equation becomes

$$
\frac{\partial S_{k}}{\partial \vec{\phi}}=\vec{\phi}-\left(p \otimes^{k} K(g)+(1-p) \otimes^{k} K(1 / g)\right) \vec{\phi}^{2}=0
$$

the matrices $K(g), K(1 / g)$ differ only in the sign of the eigenvalue $\tanh (\beta)$, so it is still possible to derive an explicit expression for the Hessian, which in this case is

$$
\operatorname{det}\left(\frac{\partial^{2} S_{k}}{\partial \vec{\phi}^{2}}\right)=\prod_{m=0}^{k}\left[2 \tanh (\beta)^{m}\left(p+(-1)^{m}(1-p)\right)-1\right]^{\left(\begin{array}{c}
k \\
m
\end{array}\right)} .
$$

We thus reproduce the values of $\beta_{M}$ and $\beta_{S G}$ calculated from equs.(3,4) for general $p$ as the first two bifurcation points. On $\phi^{z}$ graphs we replace $2 \rightarrow z-1$, which is still in accordance with equs. $(3,4)$. As all even bifurcation points are independent of $p$, we predict the $p$ independence of the spin glass transition point, as in the more standard approaches.

Equs. $(3,4)$ are not restricted to distributions of the form $P(J)=p \delta(J-1)+(1-p) \delta(J+1)$, but we can extend the approach here to accommodate this by making the observation that $K\left(g_{1}\right)$ will commute with another $K\left(g_{2}\right)$ for any $g_{1}, g_{2}$. Thinking of a given distribution (a gaussian, say) as a weighted sum of delta functions over $K$ 's with different values of $g$, we can still diagonalize all of the terms in the sum simultaneously and arrive at an expression of the form

$$
\operatorname{det}\left(\frac{\partial^{2} S_{k}}{\partial \vec{\phi}^{2}}\right)=\prod_{m=0}^{k}\left[2 \int P(J) \tanh (\beta J)^{m} d J-1\right]^{\left(\begin{array}{c}
k \\
m
\end{array}\right)}=0 .
$$

for the bifurcation equation.

Although the continuous transition temperatures $\beta_{m}$ are independent of the number of replicas, the multiplicity of solutions (ie low temperature phases) that bifurcate at a given point are not. There are $k(k-1) / 2$ solutions bifurcating at the spin glass transition temperature $\beta_{2}$, so just as in the infinite range model there is a change in the nature of the solutions to the saddle point equation when $k<1$ and the number of solutions is formally negative. The classification of the spin glass solutions in the current case is thus clearly closely related to the parametrization of the matrix $Q_{a b}$ that appears in the saddle point equations of the infinite range model [16]. 
It is also possible to consider Potts spins on a Bethe lattice [7, 8]. We take Hamiltonian in this case to be

$$
H=2 \beta \sum_{<i j>} \delta_{\sigma_{i}, \sigma_{j}}
$$

where the spins $\sigma_{i}$ can now take on $Q$ values 1 . The critical temperatures for ferromagnetic and spin glass ordering calculated in [7] give, in the style of equs.(1).21,

$$
\int_{-\infty}^{\infty} P(J)\left(\frac{\exp \left(2 \beta_{F M} J\right)-1}{\exp \left(2 \beta_{F M} J\right)+Q-1}\right) d J=\frac{1}{z-1}
$$

and

$$
\int_{-\infty}^{\infty} P(J)\left(\frac{\exp \left(2 \beta_{S G} J\right)-1}{\exp \left(2 \beta_{S G} J\right)+Q-1}\right)^{2} d J=\frac{1}{z-1}
$$

for $Q$ state Potts models. If we consider the ferromagnet, $P(J)=\delta(J-1)$, we find that $\exp \left(2 \beta_{F M}\right)=$ $(z+Q-2) /(z-2)$. There is, however, a parallel here with the Ising spin glass calculations. It was noted in [8] that this temperature is actually a spinodal point, for $Q>2$ the Potts ferromagnet undergoes a first order transition before reaching this point. Arguing, as in the Ising case, that a random graph looks locally like the Bethe lattice we would expect the various critical temperatures and spinodal points still to apply on $\phi^{z}$ graphs.

It is also possible to solve the saddle point equations explicitly to avoid relying on this analogy. For the 3 -state Potts model with action

$$
S=\frac{1}{2}\left(\phi_{a}^{2}+\phi_{b}^{2}+\phi_{c}^{2}\right)-c\left(\phi_{a} \phi_{b}+\phi_{a} \phi_{c}+\phi_{b} \phi_{c}\right)-\frac{1}{3}\left(\phi_{a}^{3}+\phi_{b}^{3}+\phi_{c}^{3}\right)
$$

this gives high and low temperature solutions 5

$$
\begin{aligned}
\phi_{a, b, c} & =1-2 c \\
\phi_{a, b} & =\frac{1+\sqrt{1-4 c-4 c^{2}}}{2} \\
\phi_{c} & =\frac{1+2 c-\sqrt{1-4 c-4 c^{2}}}{2}
\end{aligned}
$$

where $c=1 /(g+1)$. This gives a changeover in behaviour at $c=1 / 5$, ie $g=4$, which agrees with the value from equ.(32) and thus picks out the spinodal point. Similarly, solving the 4 -state Potts model equations gives the solutions

$$
\begin{aligned}
\phi_{a, b, c, d} & =1-3 c ; \\
\phi_{a, b, c} & =\frac{1+3 c-\sqrt{1-6 c-3 c^{2}}}{2}, \\
\phi_{d} & =\frac{1-c+\sqrt{1-6 c-3 c^{2}}}{2}
\end{aligned}
$$

where $c=1 /(g+2)$ here. The changeover here is at $g=5$, again apparently at the spinodal point. It would be interesting to carry out a simulation to check the nature of the transition in these ferromagnetic models.

Analysis of the Potts spin glass saddle point equations follows a similar tack to those of the Ising model. The caveats about missing potential first order transitions at finite $k$ still, of course, apply. If we

\footnotetext{
${ }^{4}$ We have adopted a different normalization for the Potts Hamiltonian from []], taking a factor of 2 rather than $Q$ in front, for consistency with

${ }^{5}$ We gave these, and those for the 4-state model, incorrectly in [1].
} 
take the three state Potts model as an example $K$ is replaced by the $3 \times 3$ matrix $L(g)$ 6

$$
L_{a b}=\left(\begin{array}{ccc}
1 & \frac{1}{g} & \frac{1}{g} \\
\frac{1}{g} & 1 & \frac{1}{g} \\
\frac{1}{g} & \frac{1}{g} & 1
\end{array}\right)
$$

and the high temperature solution on $\phi^{3}$ graphs is $(t, t, t)$ where

$$
t=\frac{g}{g+2} \text {. }
$$

The eigenvalues of $\tilde{L}(g)=t L(g)$ are $1, \lambda, \lambda$, where $\lambda=(g-1) /(g+2)$. It is still true that $\tilde{L}\left(g_{1}\right), \tilde{L}\left(g_{2}\right)$ will commute for any $g_{1}, g_{2}$ so we can put this to good use again to derive the Hessian for general $P(J)$

$$
\operatorname{det}\left(\frac{\partial^{2} S_{k}}{\partial \vec{\phi}^{2}}\right)=\prod_{m=0}^{k}\left[2 \int P(J)\left(\frac{\exp (2 \beta J)-1}{\exp (2 \beta J)+2}\right)^{m} d J-1\right]^{\left(\begin{array}{c}
k \\
m
\end{array}\right) 2^{m}} .
$$

The multiplicity of solutions has changed because of the possibility of taking one of two $\lambda$ from each replica. The results for $\beta_{F M}$ and $\beta_{S G}$ from the Bethe lattice are thus faithfully reproduced as the first two bifurcation points.

Generalizing to the $Q$ state Potts model on $\phi^{z}$ graphs, we find

$$
t=\left(\frac{g}{g+Q-1}\right)^{1 /(z-2)}
$$

and the eigenvalues of the $Q \times Q$ matrix $\tilde{L}$ will be 1 and $Q-1 \lambda$ 's, where $\lambda=(g-1) /(g+Q-1)$. This gives the Hessian

$$
\operatorname{det}\left(\frac{\partial^{2} S_{k}}{\partial \vec{\phi}^{2}}\right)=\prod_{m=0}^{k}\left[(z-1) \int P(J)\left(\frac{\exp (2 \beta J)-1}{\exp (2 \beta J)+Q-1}\right)^{m} d J-1\right]^{\left(\begin{array}{l}
k \\
m
\end{array}\right)(Q-1)^{m}}
$$

which the reader will be pleased to hear is the last elaboration of the bifurcation equation that we consider. The $Q=2$ case reproduces the Ising results derived earlier.

Given the accessibility of mean-field results on random graphs, especially as one is not forced to take infinite range interactions or deal with the boundary difficulties of the Bethe lattice, the lack of simulational effort is rather surprising. As the new viewpoint offered by regarding the random graphs as Feynman diagrams offers the possibility of attacking various questions, such as the nature of replica symmetry breaking on random graphs, from a different angle, we thought it important to continue the simulations of [1] for the Ising and $Q$ state Potts spin glasses on $\phi^{3}$ graphs to check carefully the agreement with the known results for quantities such as the transition temperatures and critical exponents and to gain a thorough understanding of the numerics before going on to investigate less well understood aspects. Our numerical methods are largely similar to those of [1], so we describe them only briefly in the next section and concentrate on the results of the simulations.

\section{Simulations: Generalities and Ising Model}

In all of the simulations we report here we generated around 100 different Feynman diagrams at each of the sizes we simulated, 100, 250, 500 and 1000 vertices. The quenched bond distribution on each graph was $P(J)=p \delta(J-1)+(1-p) \delta(J+1)$, with $p$ taken to be 0.5 (the " $\pm J$ " distribution) unless specified otherwise. Various massively parallel processors were used to run the simulations of the different graphs simultaneously and perform the quenched averaging over the graphs in situ. Again,

\footnotetext{
${ }^{6}$ This definition of $L$, which is the simplest from the point of view of the saddle point equations, involves a rescaling with respect to equ.(34) which accounts for the different between $t$ and the high temperature solution in equ. 35 .
} 
in the interests of simplicity and reliability (and as in [1]) we used only a metropolis algorithm with simulated annealing rather than some of the more advanced algorithms developed recently. At each $\beta$ value simulated we carried out 500,000 simulated annealing sweeps, followed by 500,000 production sweeps with a measurement every tenth sweep. Each sweep consisted of a complete metropolis update of the lattice. Our strategy for extracting the critical exponents will be to use Binder's cumulant for the overlap, defined below, to extract an estimate for $\beta_{S G}$ and the combination $\nu d$ and then to look at the finite size scaling of other quantities to extract further exponents.

In the Ising model the spin glass transition temperature is found in simulations by putting two Ising replicas on each graph with spins $\sigma_{i}, \tau_{i}$ to measure the overlap, which is the order parameter for the spin glass transition

$$
q=\frac{1}{n} \sum_{i} \sigma_{i} \tau_{i}
$$

The Binder's cumulant for the overlap is then defined in an analogous fashion to the Binder's cumulant for the magnetization in a ferromagnetic transition

$$
U_{s g}=\frac{\left[\left\langle q^{4}\right\rangle\right]}{\left[\left\langle q^{2}\right\rangle\right]^{2}}
$$

where \langle\rangle denote thermal averages and [ ] disorder averages. The plots of $U_{s g}$ for differently sized graphs are expected to cross at $\beta_{S G}$. In the course of all the simulations the overlap distribution

$$
P_{n}(q)=\left[\left\langle\delta\left(q-1 / n \sum \sigma_{i} \tau_{i}\right)\right\rangle\right]
$$

was also histogrammed. A non-trivial $P(q)$ is a strong, but not infallible, signal for a spin glass phase. It is perhaps worth remarking that the order of averages in eqn.(43) is not absolutely obvious a priori - one might have considered $\left[<q^{4}>/<q^{2}>^{2}\right.$ ], for instance. Regarding $<q^{n}>$ as moments of the distribution $P(q)$, the choice in the eqn.(43) would seem most appropriate. The alternative overall average has been considered in quantum spin glass simulations [17] on the heuristic grounds that it gives better scaling behaviour. We have carried out the scaling analysis of our simulations described below with both definitions for the Ising spin glass and found essentially identical results.

We expect the overlap to have the finite size scaling form $q \simeq n^{-\beta / \nu d}$ at any spin glass transition and the spin glass susceptibility, defined as

$$
\chi_{s g}=\frac{1}{n} \sum_{i j}\left[\left\langle\sigma_{i} \sigma_{j}\right\rangle^{2}\right]
$$

or, alternatively,

$$
\chi_{s g}=n \int q^{2} P_{n}(q) d q .
$$

to diverge as $\chi_{s g} \simeq n^{\gamma / \nu d}$. Other critical exponents such as that for the specific heat $C \simeq B+C_{0} n^{\alpha / \nu d}$ are defined in the standard manner and relations such as $\alpha=2-\nu d$ still hold good.

The mean field critical exponents are shown in the table below for an Ising $(Q=2)$ spin glass Table 1: Spin Glass exponents for Ising model

\begin{tabular}{|c|c|c|c|c|c|}
\hline$\alpha$ & $\beta$ & $\gamma$ & $\delta$ & $\nu d$ & $\eta$ \\
\hline-1 & 1 & 1 & 2 & 3 & 0 \\
\hline
\end{tabular}

We have written $\nu d$ in the table rather than $\nu$ as we cannot disentangle $\nu$ and $d$ in the infinite dimensional random graph case at hand.

We now commence the analysis of the results of the simulations proper for the Ising model. The cumulant for the overlap is plotted using both ways of carrying out the averages in Fig.1 and Fig.2, from which it is clear that the resulting crossover points are, as claimed, very similar. Fitting the crossover 
point from either figure gives $\beta_{S G}=0.88(1)$, although the overall average that gives Fig.2 is slightly noisier. This value is in good agreement with the theoretical predictions. An additional consequence of equ.(4) is that $\beta_{S G}$ should be independent of $p$ for $P(J)=p \delta(J-1)+(1-p) \delta(J+1)$. In [1] we considered the Ising antiferromagnet, which has $p=0$, and found $\beta_{S G}=-0.94(2)$ by direct observation of cumulant crossing, but we also found $\beta_{S G}=-0.88$ (2) from extrapolation of the specific heat peak. These results are thus only marginally compatible with the $p$ independence. As a further check we simulated a complete set of graphs with $p=0.3$ and found that the cumulants were identical within the errors to the $p=0.5$ values, this giving the same $\beta_{S G}$. The slight discrepancy at $p=0$ may well be due to the poorer statistics we have for these runs.

Further analysis of the cumulants allows us to extract $\nu d$ by looking at the scaling of the maximum slope of the cumulant with the number of vertices $n$.

$$
\max \left(\frac{d U}{d \beta}\right) \simeq n^{\frac{1}{\nu d}}
$$

We find that $\nu d=2.9(1)$ from the data in Fig.1 and $\nu d=3.0(2)$ from that in Fig.2. The simulations in [1] found the compatible value of $\nu d=2.8(2)$ for $p=0$ and all are in agreement with the mean-field value of $\alpha=-1$ for the specific heat critical exponent, deduced from $\alpha=2-\nu d$. A cusp in the specific heat $C$, rather than a divergence, is indicated by this negative value of $\alpha$ in the finite size scaling relation

$$
C \simeq B+C_{0} n^{\alpha / \nu d}
$$

The cusp can clearly be seen in Fig.3. A direct fit to the scaling of the specific heat as in eqn.(48) is not particularly instructive, as the extra adjustable constant $B$ allows for a very good fit to the value $\alpha / \nu d=-1 / 3$, but it is at least consistent with the value emerging from the cumulant analysis.

Turning now to the expected scaling of the overlap, we find respectable agreement with the finite size scaling relation

$$
q \simeq n^{-\beta / \nu d}
$$

with $\beta / \nu d$ calculated to be $0.30(1)$ by extrapolating to the pseudocritical value of $\beta_{S G}=0.88$. Having considered the first moment of $P(q)$ with $q$, we now move on to what is effectively the second moment of the $P(q)$ with $\chi_{s g}$. In Fig.4 we see the expected divergence, and a fit to the finite size scaling relation

$$
\chi_{s g} \simeq n^{\gamma / \nu d}
$$

gives $\gamma / \nu d=0.34(2)$, in agreement with the mean field value of $\gamma=1$. No divergence is expected in the linear susceptibility

$$
\chi_{M}=\frac{d M}{d H}
$$

and, as is clear from Fig.5, none is seen. We have not measured the response of the model to an external field, nor attempted to fit the correlation functions at the critical temperature, so we have no estimates for $\delta$ and $\eta$. However, it is clear from the above results that the model is giving mean-field like behaviour for the spin glass transition that is observed.

The results for the specific heat and the susceptibilities reported here for the $\pm J$ spin glass are essentially identical to those in [1] for the Ising antiferromagnet, which can be taken as a further confirmation of the $p$ independence of the spin glass transition. Looking directly at the histograms of $P(q)$ themselves in the spin glass phase for $p=0$ (the antiferromagnet) and $p=0.3,0.5$ (the models simulated here) does show some differences as can be seen in Fig.6. However, the determination of the critical point depends on the scaling of the moments of $P(q)$ around the transition point at $\beta=0.8814 \ldots$ and it is here we expect to find $p$ independence. This is indeed what we see at $\beta=0.9$ in Fig.7 - the histograms are essentially identical for $p=0.3,0.5$. There is a slight discrepancy still for $p=0$, which is a reflection of the less convincing scaling analysis in [1] that determined the critical point in the case of the pure antiferromagnet. We have shown only the $P(q)$ for graphs with 500 points in the figures for clarity, the results for other graph sizes are identical. 


\section{Simulations: Potts Models}

For $Q$-state Potts models the overlap may be defined as

$$
q=\frac{1}{n} \sum_{i=1}^{n}\left(Q \delta_{\sigma_{i}, \tau_{i}}-1\right)
$$

which is arranged to be zero in the uncorrelated case. The cumulants and $P(q)$ may then be defined in an analogous fashion to the Ising model. There is, however, no longer the $q \rightarrow-q$ symmetry in $P(q)$ that is present in the Ising spin glass for $Q \geq 3$ state Potts models. In all of the simulations reported here we took $P(J)=1 / 2(\delta(J-1)+\delta(J+1))$. We simulated lattices of size $100,250,500$ and 1000 for the $Q=3,4$ state models, but only the three smaller lattice sizes for $Q=10,50$. The statistics and annealing schedules were identical to the Ising model simulations. We are principally interested in observing the qualitative features of the various Potts models, so our analysis concentrates less on serious finite size scaling to extract the exponents than the Ising results.

Mean field theory (in the infinite range model) suggests that $Q \geq 3$ Potts glasses are rather different in behaviour from the Ising spin glass as there is no longer continuous replica symmetry breaking. For $Q=3,4$ there are two consecutive transition temperatures $\beta_{S G}$ and $\beta_{S G 2}$ to different glass phases and for $Q>4$ the first has a discontinuity in the overlap $q$. For $\beta_{S G 2}>\beta>\beta_{S G} P(q)$ consists of a delta function at $q=0$ and another delta function at finite $q$ with no continuous features whereas for $\beta>\beta_{S G 2}$ the delta function at finite $q$ splits in two with a continuous distribution between. The values of the transition temperature given by equ. $(33,41)$ are for $\beta_{S G}$. Some features of the solution differ on the Bethe lattice [7], in particular an Ising-like solution appears to exist for coordination number three with a $\pm J$ bond distribution, and the $\phi^{3}$ random graph model might be expected to behave in this fashion too.

If we take the 3 and 4 state Potts models first, we can look at the cumulant crossing in a similar manner to the Ising model to attempt to pinpoint the phase transition. It is possible to extract crossing points at $\beta=1.5(1)$ for $Q=3$ and $\beta=2.0(1)$ for $Q=4$, which are in agreement with the values calculated from equ.(33) shown below in Table.2. Our data points for the $Q=10,50$ state models are rather sparser but the cumulant crossing appears to become more clear cut with increasing $Q$, which offsets this.

\begin{tabular}{|c|c|c|c|c|c|}
\hline$Q$ & 2 & 3 & 4 & 10 & 50 \\
\hline$\beta_{S G}$ & 0.8814 & 1.5824 & 2.1226 & 3.6914 & 6.1943 \\
\hline measured & $0.88(1)$ & $1.5(1)$ & $2.0(1)$ & $3.6(4)$ & $6.1(1)$ \\
\hline
\end{tabular}

Table 2: $\beta_{S G}$ for various $Q$ state Potts models on $\phi^{3}$ graphs.

The quoted errors in the table are the most conservative choice - the largest difference between the crossing points for the various lattice sizes - apart from $Q=3$ where the $N=100$ data was dropped, as it failed to cross with the other lattice sizes.

We next look at the qualitative features of the low temperature phase in the various $P(q)$ to see if the mean field theory expectations are borne out. With our definitions, the histograms of $P(q)$ range from -1 to $Q-1$ for a $Q$ state Potts model with the origin at zero and roughly half of the probability density in the range $0 \ldots(Q-1)$, so we have plotted only $0 \ldots(Q-1)$, normalized to the range $0 \ldots 1$ for clarity in all our figures In all of the models the high-temperature $P(q)$ look like skewed gaussians centred on the origin, whose sharpness increases with graph size indicating delta-function-like behaviour in the continuum limit. In Fig. 8 we have plotted $P(q)$ for the three state Potts model for $\beta=2.2\left(>\beta_{S G}\right)$. This is clearly different from the Ising model: there is a strong peak at the origin that is increasing with graph size as well as smaller bump at around $q=0.7$ which is also increasing with graph size and which might tentatively be identified with another peak in the continuum limit. In [1] simulations of the Potts

\footnotetext{
${ }^{7}$ It is also possible to extract a positive scalar order parameter for the spin glass phase by going to a simplex representation for the Potts spins and using the radial component of the overlap matrix, but we stick with the canonical definition here.
} 
antiferromagnet, where no spin glass phase is expected, saw no signs of the structure at $q=0.7$, with $P(q)$ remaining resolutely centred on the origin. Although the very large autocorrelation times that are encountered for $\beta>\beta_{S G}$ make the interpretation of the low temperature results a dangerous business we can press on deeper into the low temperature region to look at $P(q)$ there and see if there is any evidence for the second transition. $P(q)$ is plotted at $\beta=3.5$ for the same three lattice sizes in Fig.9, where we can see that there does, indeed, appear to be a second peak developing away from the origin at $q=0.2$ as well as the bump at $q=0.7$.

The qualitative features of the above results are preserved in the $Q=4,10,50$ state models that we also simulated: the histogram of $P(q)$ broadens from a sharp peak at the origin at around $\beta_{S G}$ and develops a secondary bump or shoulder. There is some evidence of further structure developing for even larger $\beta$. From the numerical evidence it would thus appear that the $Q=3$ Potts spin glass with a $\pm J$ distribution of bonds lies above the critical $Q$ value that separates Ising and Potts spin glass behaviour, whereas the Bethe lattice calculation in [7] supports the opposite conclusion, although on the relatively small lattices simulated here it is difficult to distinguish between features that will be sharp in the continuum limit and continuous parts of $P(q)$. A more detailed analysis of the saddle point equations may shed some light on this question analytically. For completeness in Fig. 10 we show $P(q)$ for the $Q=4$ model at $\beta=4.4\left(>\beta_{S G}\right)$ to demonstrate the similarity with the $Q=3$ results.

The fits to the maxima of the Binder's cumulant to extract $\nu d$ are rather poor for all the higher state Potts models, but the specific heat curves for $Q=3,4$ are similar in form to the Ising model, as can be seen for the $Q=4$ Potts model in Fig.11, indicating the continued presence of a cusp rather than a divergence. We therefore do not have a reliable value of $\nu d$ to feed into the finite size scaling relations, although the cusp suggests that $\nu d \simeq 3$, at least for $Q=3,4$. Nonetheless, we can still fit to find the combinations $\beta / \nu d$ and $\gamma / \nu d$ at the estimated critical points for the various models. This gives $\beta / \nu d=0.28(2), 0.31(5)$ for the $Q=3,4$ state models respectively. The mean field theory suggests that $q$ becomes discontinuous at $\beta_{S G}$ for $Q>4$. With the smaller lattices that we have for $Q=10,50$ this effect, if it exists, is masked by finite size rounding but attempting to fit $\beta / \nu d$ does give larger values and much poorer fits than for $Q=3,4$ such as $0.45(5)$ for $Q=10$. This could be construed as providing some evidence for a discontinuity. The data allowed fits to $\gamma / \nu d$ only for the $Q=3,4$ models, where it gave $0.36(2), 0.40(7)$ respectively.

In summary, all the Potts $P(q)$ are clearly qualitatively different from the Ising model, even though the two exponents $\beta / \nu d, \gamma / \nu d$ we fitted for $Q=3,4$ are roughly similar. There is not sufficient data in the simulations reported here to reliably distinguish a discontinuous transition for $Q>4$, though there are certainly indications (the fits to $\beta / \nu d$ ) that this is the case. In all the cases, however, the values extracted for the spin glass transition temperature by analysis of Binder's cumulant are in agreement with the predictions.

\section{Conclusions}

The novel analytical approach offered by using techniques borrowed from matrix models in the manner of [9] to look at spin models on random graphs allows one to rederive results for transition temperatures and order parameters that are less transparent in previous replica calculations, or only arrived at by analogy with the Bethe lattice. Indeed, results from bifurcation theory enabled us to calculate the Hessians for bifurcations from the symmetric solution (which may not be dominant all the way to the bifurcation point, as is seen in the $k=3,4$.. solutions) in $k$ replica Ising or Potts models for any $k$ and $P(J)$. The number of putative spin glass solutions bifurcating at $\beta_{2}$ appeared to behave as in the the infinite range model. In essence the investigation of a spin glass on a $z$-regular random graph is reduced to looking at the solutions to the equation

$$
\begin{aligned}
\vec{\phi} \quad & \int P(J) \otimes^{k} K d J \vec{\phi}^{z-1} \\
& \lim k \rightarrow 0 .
\end{aligned}
$$

Further work along these lines is clearly both desirable and possible for the Ising and other Potts models, with the nature of replica symmetry breaking in this short range (but still mean field) model being perhaps 
the most important question. We have made no attempt here to follow the various solution branches that bifurcate at $\beta_{2}$, for instance, which would shed light on the low temperature phase, particularly if calculations for arbitrary $k$ were still possible. An understanding of the role of the first order solutions that appear for $k>2$ and their failure to influence the $k \rightarrow 0$ limit is also still missing. We emphasize again that the transitions in the thin graph model appear to be identical with those in the Ising replica magnet where it is the $k=2$ transition that marks the boundary between first order transitions and the behaviour seen in the quenched model at $k=0$.

We investigated the models numerically in some detail. Quantitatively, transition temperatures were in agreement with those calculated for all the models. The extraction of the critical exponents for the Ising model showed clear mean field behaviour, and the $p$-independence of the spin glass transition temperature was also apparent. Qualitatively, the $P(q)$ measured in the various models backed up the mean field picture of the phase transition with a continuous distribution for the Ising model and sharper features for $Q>3$.

In summary, spin glasses on thin graphs offer a promising arena for the application of ideas from matrix models, large- $n$ calculations in field theory and bifurcation theory. The tensor (or near-tensor) product of the inverse propagator allows some quite general expressions to be derived for the Hessian in the saddle point equations and offers a powerful line of attack on questions such as replica symmetry breaking. As a subject for numerical simulations they offer the great advantage of mean field results with no infinite range interactions and no boundary problems.

It is worth remarking in closing that one is not limited to the mean-field theory with the current methods. It is possible to "fatten" the graphs analytically by increasing the size of the matrices in the saddle point equations $N=2,3 \ldots$ as has already been done with some success for Ising models coupled to two dimensional gravity [19], which is equivalent to looking at the models on an annealed ensemble of planar (fat)graphs. This reduces the fractal dimension of the graphs from infinity in the mean field case to more realistic values. Numerical simulations of the ferromagnetic transition [20] and possible spin glass transition [1] have already been carried out on a quenched ensemble of such planar graphs and in the spin glass case $P(q)$ still presents a mean-field-like appearance. It would be very interesting to say something analytically about the nature of the low temperature phase in such a non-mean-field spin glass.

\section{Acknowledgements}

The bulk of the simulations were carried out on the Front Range Consortium's 208-node Intel Paragon located at NOAA/FSL in Boulder. Some simulations were also performed on the Cray T3D at the Conrad Zuse Centrum in Berlin. Some of the Binder's cumulant analysis was carried out using programs written by A. Krzywicki. CFB is supported by DOE under contract DE-FG02-91ER40672, by NSF Grand Challenge Applications Group Grant ASC-9217394 and by NASA HPCC Group Grant NAG5-2218. PP is supported by EPSRC grant GR/J03466 and WJ thanks the Deutsche Forschungsgemeinschaft for a Heisenberg fellowship. CFB and DAJ were partially supported by NATO grant CRG910091.

\section{References}

[1] C. Baillie, D. A. Johnston and J-P. Kownacki, Nucl. Phys. B432 (1994) 551.

[2] L. Viana and A. Bray, J. Phys. C 18 (1985) 3037.

[3] M. Mezard and G. Parisi, Europhys. Lett. 3 (1987) 1067;

I. Kanter and H. Sompolinsky, Phys. Rev. Lett. 58 (1987) 164;

K Wong and D. Sherrington, J. Phys. A20 (1987) L793;

K Wong and D. Sherrington, J. Phys. A21 (1988) L459;

C. de Dominicis and Y. Goldschmidt, J. Phys. A22 (1989) L775;

C. de Dominicis and Y. Goldschmidt, Phys. Rev. B41 (1990) 2184;

P-Y Lai and Y. Goldschmidt, J. Phys. A23 (1990) 399. 
[4] N. Persky, I. Kanter and S. Solomon, "Cluster Dynamics for Randomly Frustrated Systems with Finite Connectivity", Racah Institute preprint, to appear in Phys. Rev. Lett.

[5] H. A. Bethe, Proc. Roy. Soc. A 150 (1935) 552;

C. Domb, Advan. Phys. 9 (1960) 145;

T. P. Eggarter, Phys. Rev. B9 (1974) 2989;

E. Muller-Hartmann and J. Zittartz, Phys. Rev. Lett. 33 (1974) 893.

[6] S. Inawashiro and S. Katsura, Physica 100A (1980) 24;

S. Katsura, S. Inawashiro and S. Fujiki, Physica 99A (1979) 193;

S. Katsura, Physica 104A (1980) 333, ibid 141A (1987)556;

S. Katsura and S. Fujiki, J. Phys. C12 (1979) 1087;

D. Thouless, Phys. Rev. Lett. 56 (1986) 1082;

J. Chayes, L. Chayes, P Sethna and D. Thouless, Comm. Math Phys. 106 (1986) 41;

P. Mottishaw, Europhys. Lett. 4 (1987) 333;

K Wong and D. Sherrington, J. Phys. A20 (1987) L785.

[7] Y. Goldschmidt, Europhys. Lett. 6 (1988) 7.

[8] F. Peruggi, J. Phys. A16 (1983) L713.

F. Peruggi, F. di Liberto and G. Monroy, J. Phys. A16 (1983) 811.

[9] C. Bachas, C. de Calan and P. Petropoulos, J. Phys. A27 (1994) 6121.

[10] E. Brezin, C. Itzykson, G. Parisi and J.B. Zuber, Commun. Math. Phys. 59 (1978) 35;

M.L. Mehta, Commun. Math. Phys. 79 (1981) 327.

[11] For a review see, J. Ambjorn, "Quantization of Geometry" Les Houches 1994, hep-th/9411179.

[12] J. Le Guillou and J. Zinn-Justin (editors), "Large Order Behaviour of Perturbation Theory", Amsterdam: North Holland (1989).

[13] B. Derrida, Phys. Rev. Lett 4579 (1980);

Phys. Rev. B24 2613 (1981).

[14] D. Sherrington, J. Phys. A13 637 (1980);

R. Penney, A. Coolen and D. Sherrington, J. Phys. A26 3681 (1993).

[15] M. Golubitsky and D. Shaeffer, "Singularities and Groups in Bifurcation Theory", Springer Verlag, New York (1985).

[16] S. Kirkpatrick and D. Sherrington, Phys. Rev. B17 4384 (1978);

G. Parisi, Phys. Rev. Lett. 501946 (1983).

[17] M. Guo, R. Bhatt and D. Huse, Phys. Rev. Lett. 724137 (1994);

H. Rieger and A. Young, Phys. Rev. Lett. 724141 (1994).

[18] D. Gross, I. Kanter and H. Sompolinsky, Phys. Rev. Lett. 55 (1985) 304.

[19] E. Brezin and S. Hikami, Phys. Lett. B283 203 (1992);

E. Brezin and S. Hikami, ibid B295 209 (1992);

S. Hikami, ibid 305327 (1993);

S. Hikami, Physica A204 290 (1994).

[20] D. Johnston, Phys. Lett. B277 (1992) 405;

C. Baillie, K. Hawick and D. Johnston, Phys. Lett. B328 (1994) 251.

Figure Captions 
Fig. 1 The crossover in Binder's cumulant for the overlap in the Ising spin glass calculated with individual disorder averages. Only the 250,500 and 1000 lattice data is plotted for clarity. The lines are drawn only to guide the eye and are not the best fit curves used to determine the crossing points.

Fig. 2 The crossover in Binder's cumulant for the overlap calculated with an overall average. Again, only the 250,500 and 1000 lattice data is plotted and the lines are to guide the eye only.

Fig. 3 The specific heat for the Ising spin glass.

Fig. 4 The spin glass susceptibility $\chi_{s g}$.

Fig. 5 The linear susceptibility $\chi_{M}$.

Fig. $6 P(q)$ vs $q$ for $p=0,0.3,0.5$ on graphs of 500 vertices at $\beta=1.2$, deep in the spin glass phase.

Fig. $7 P(q)$ vs $q$ for the same $p$ on graphs of 500 vertices at $\beta=0.9$, close to $\beta_{S G}$. The histogram for $p=0$, which deviates slightly, is labelled.

Fig.8 $P(q)$ vs $q$ for the three state Potts model at $\beta=2.2$. Data from graphs with 500 and 1000 vertices are plotted.

Fig.9 $P(q)$ vs $q$ for the three state Potts model at $\beta=3.5$. Data from graphs with 500 and 1000 vertices are plotted.

Fig.10 $P(q)$ vs $q$ for the four state Potts model at $\beta=4.4$. Data from graphs with 500 and 1000 vertices are plotted.

Fig.11 The specific heat for the $Q=4$ Potts spin glass. As the transition (determined from the Binder's cumulant for $q$ ) lies above the specific heat peak the values for the larger lattices are only measured on this side. 


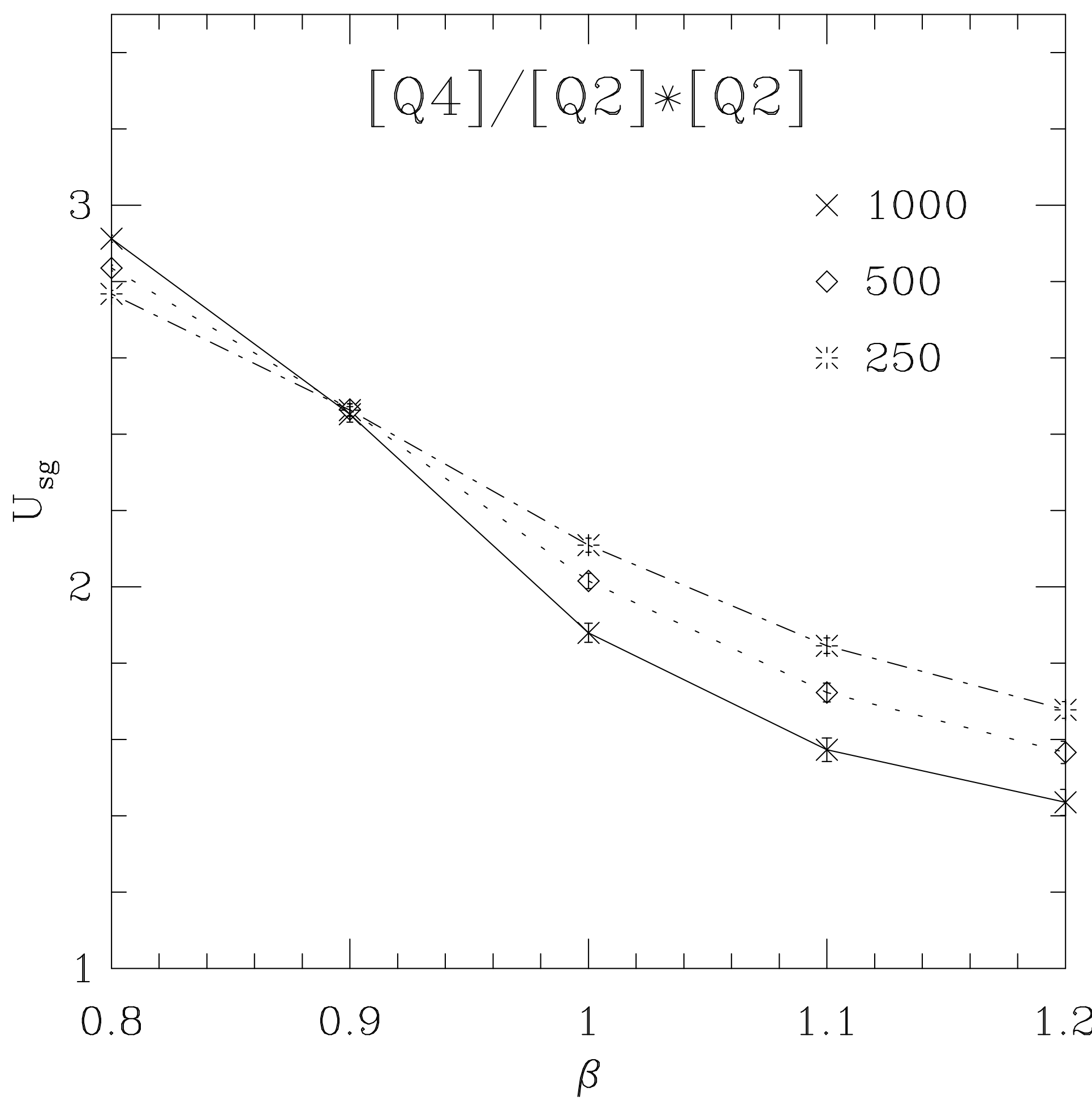




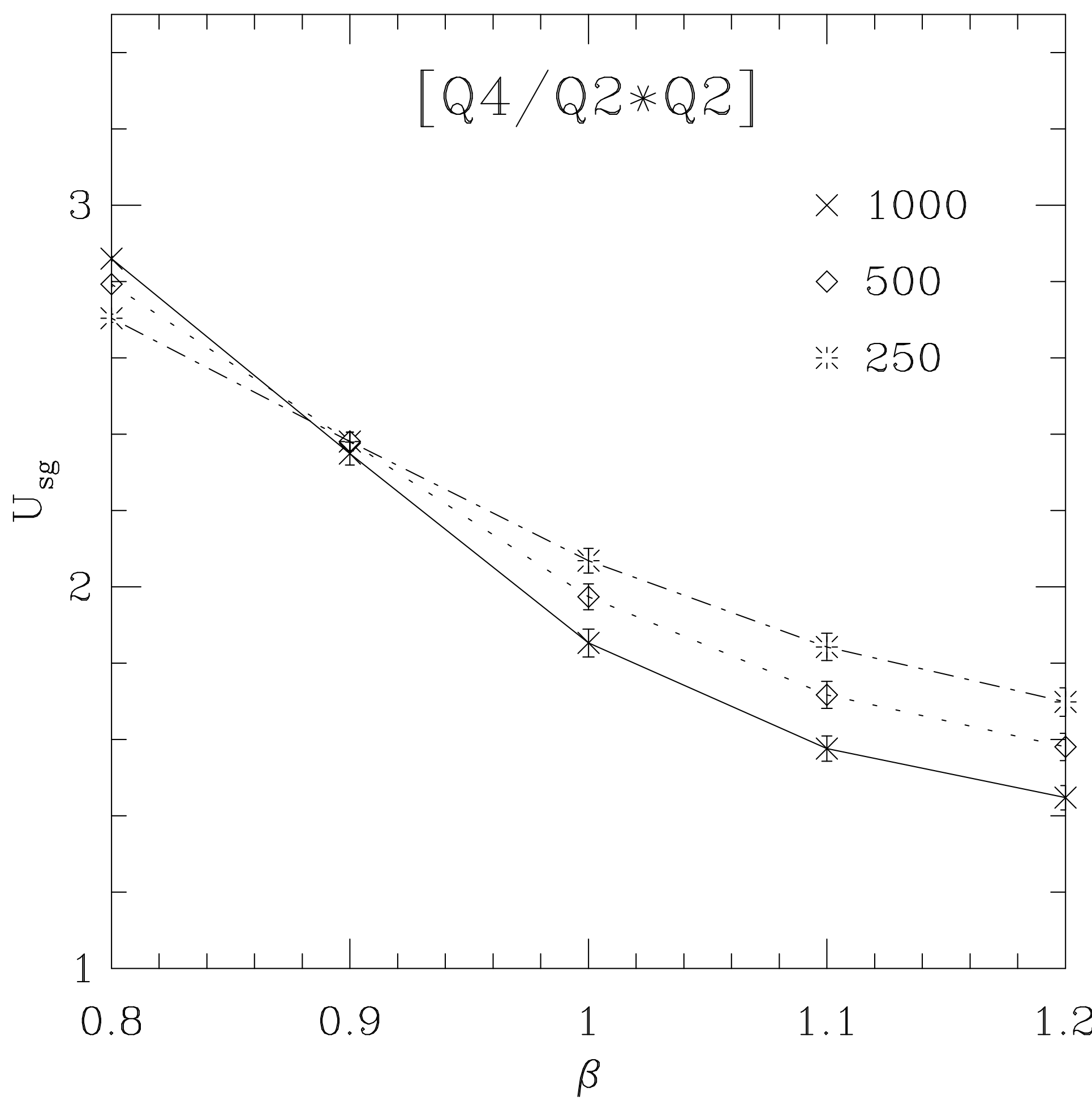




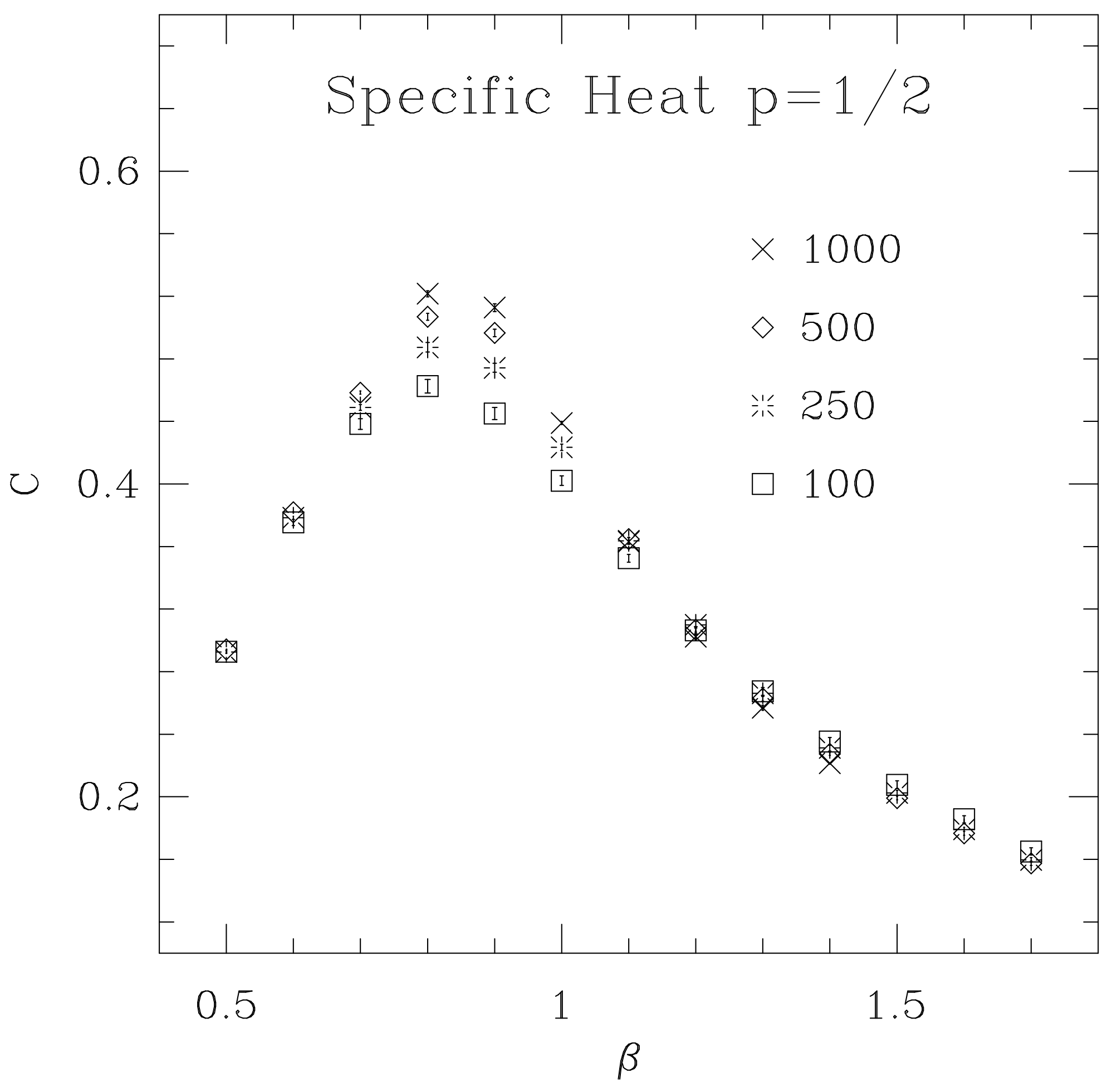




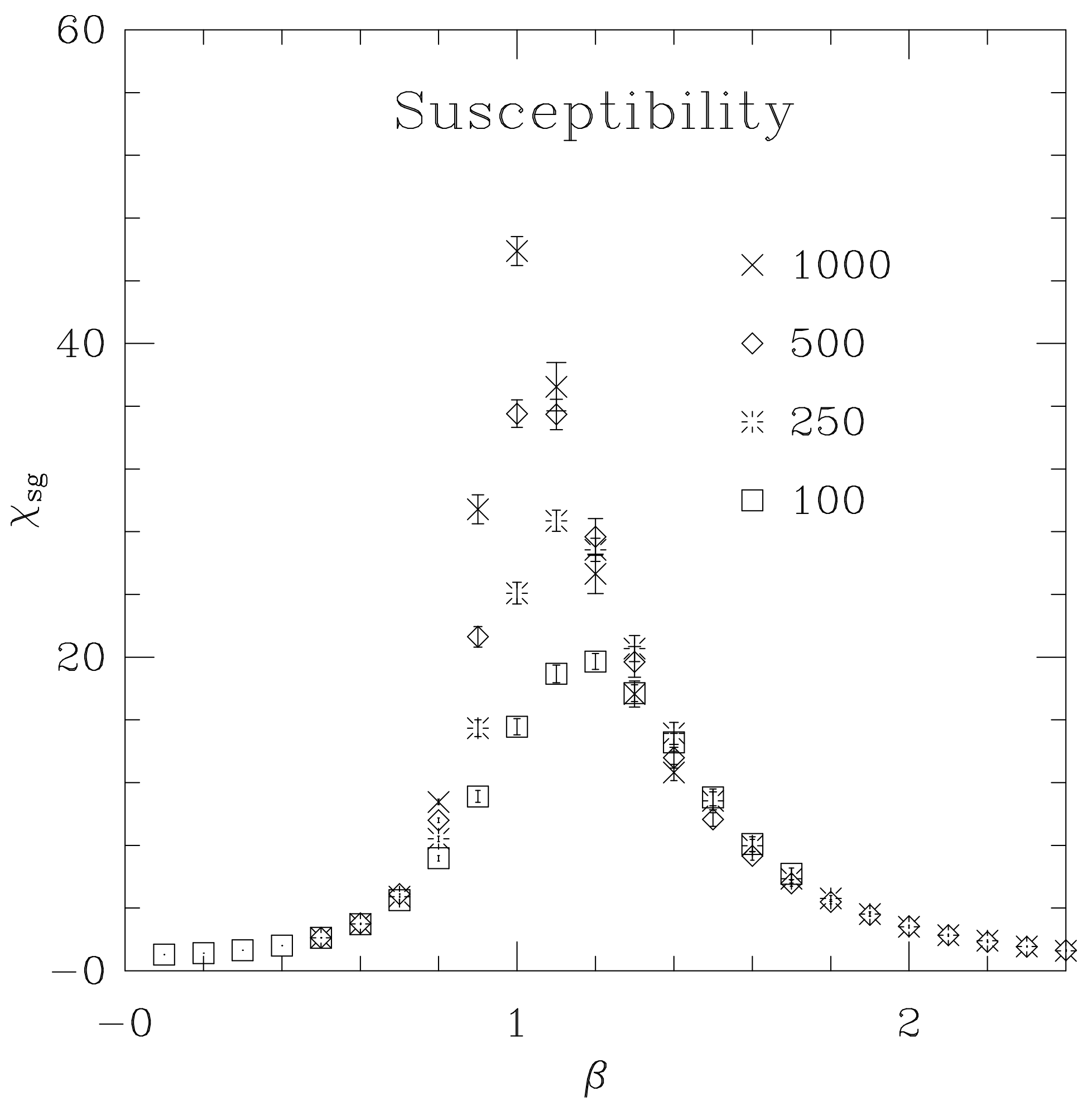




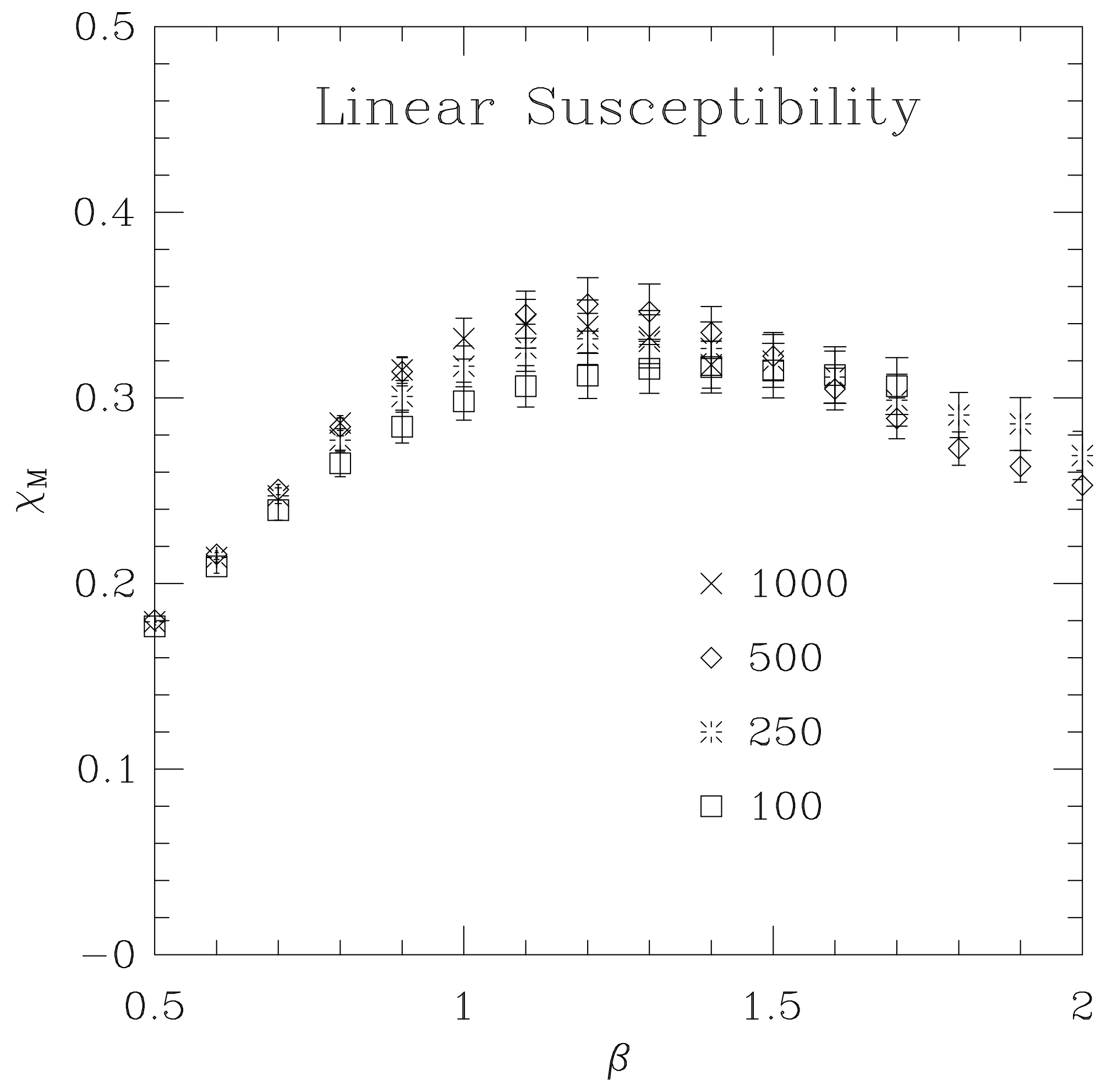




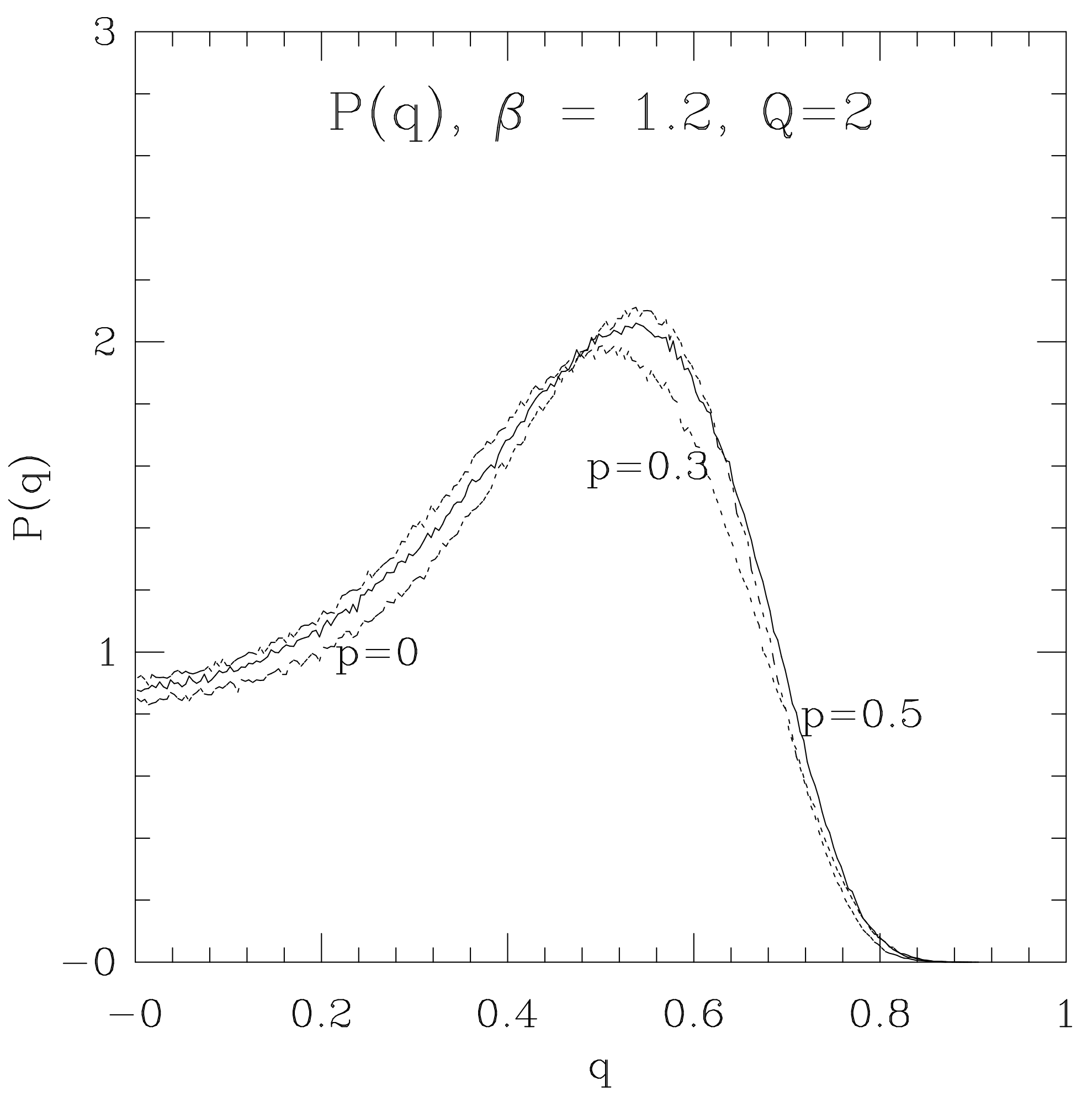




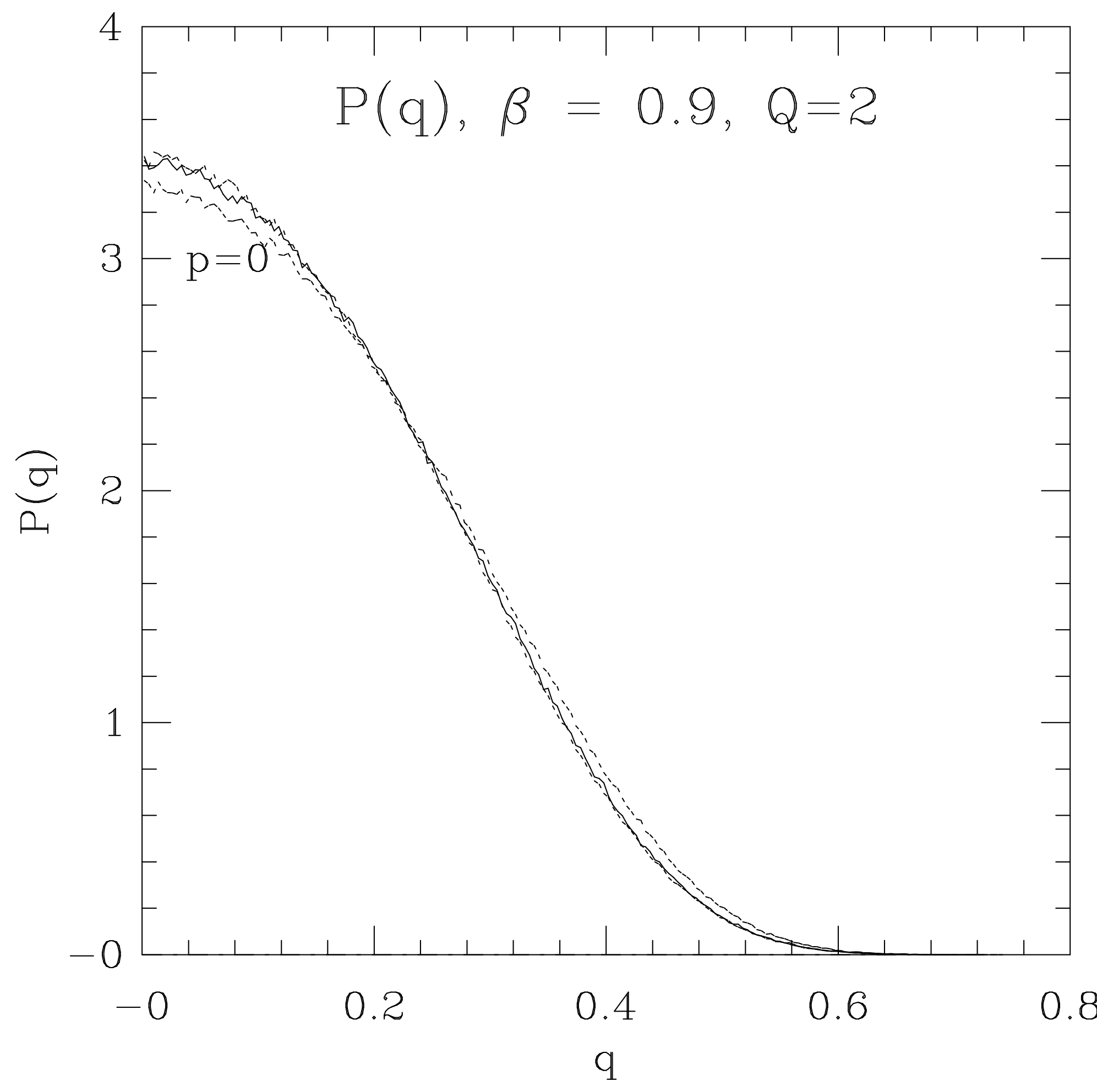




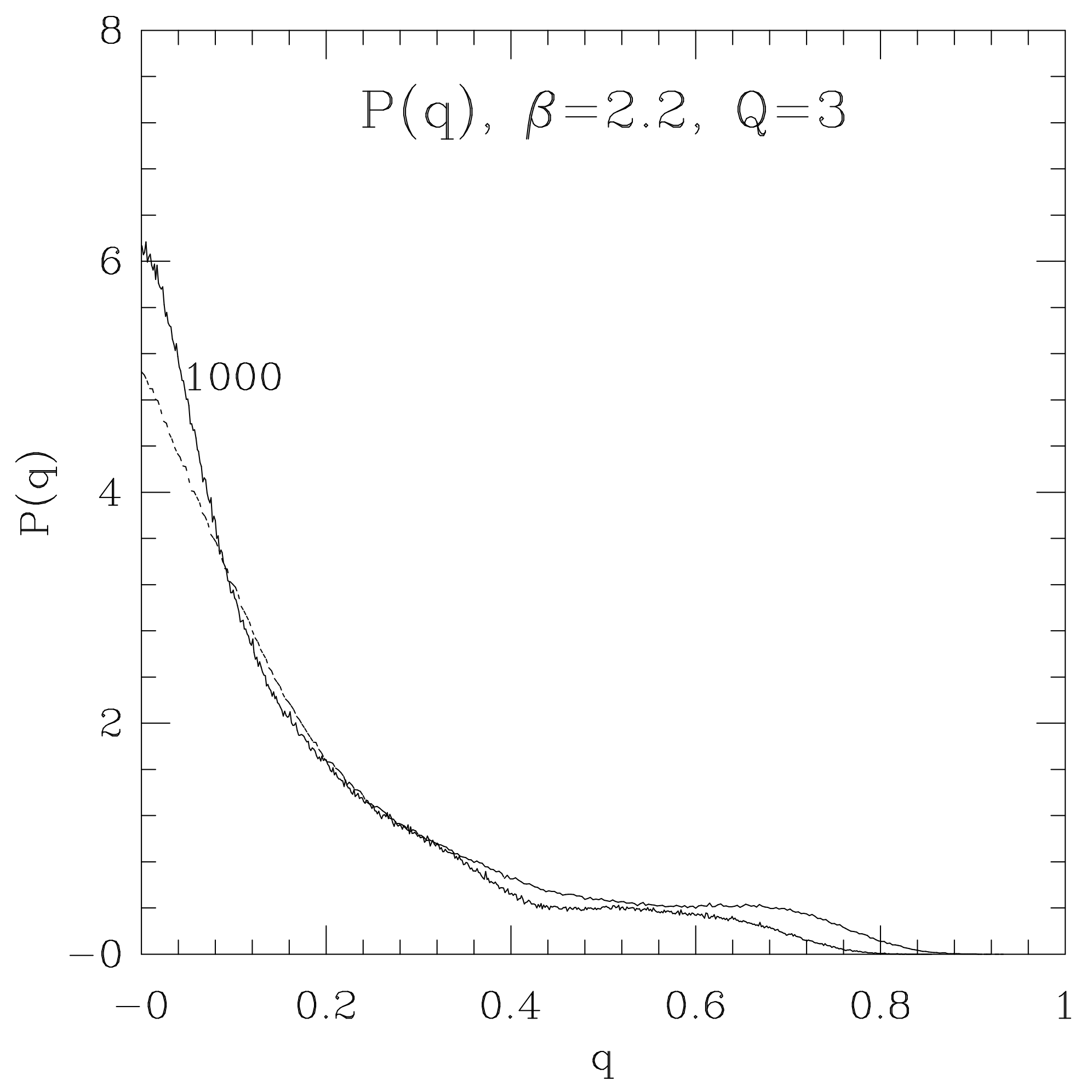




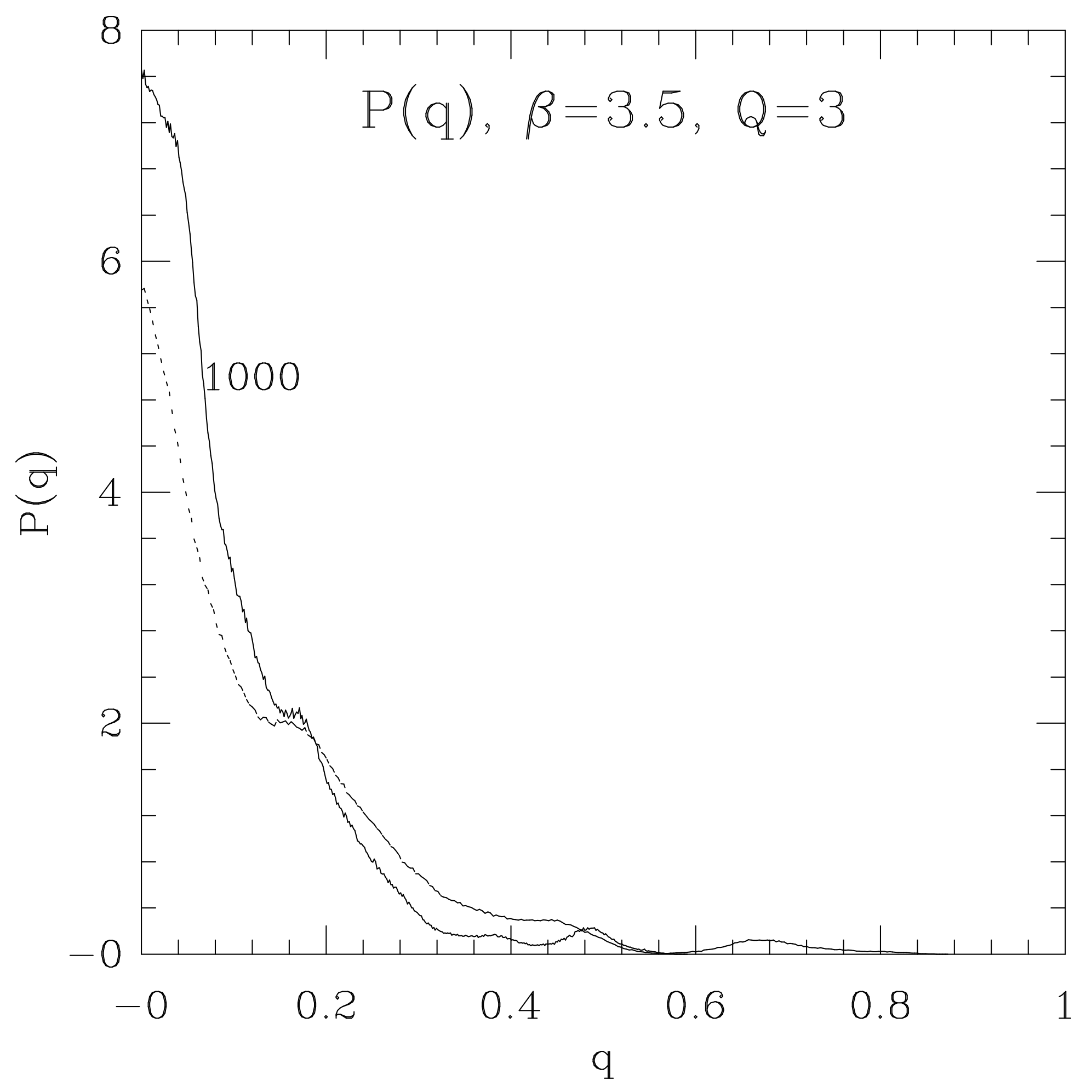




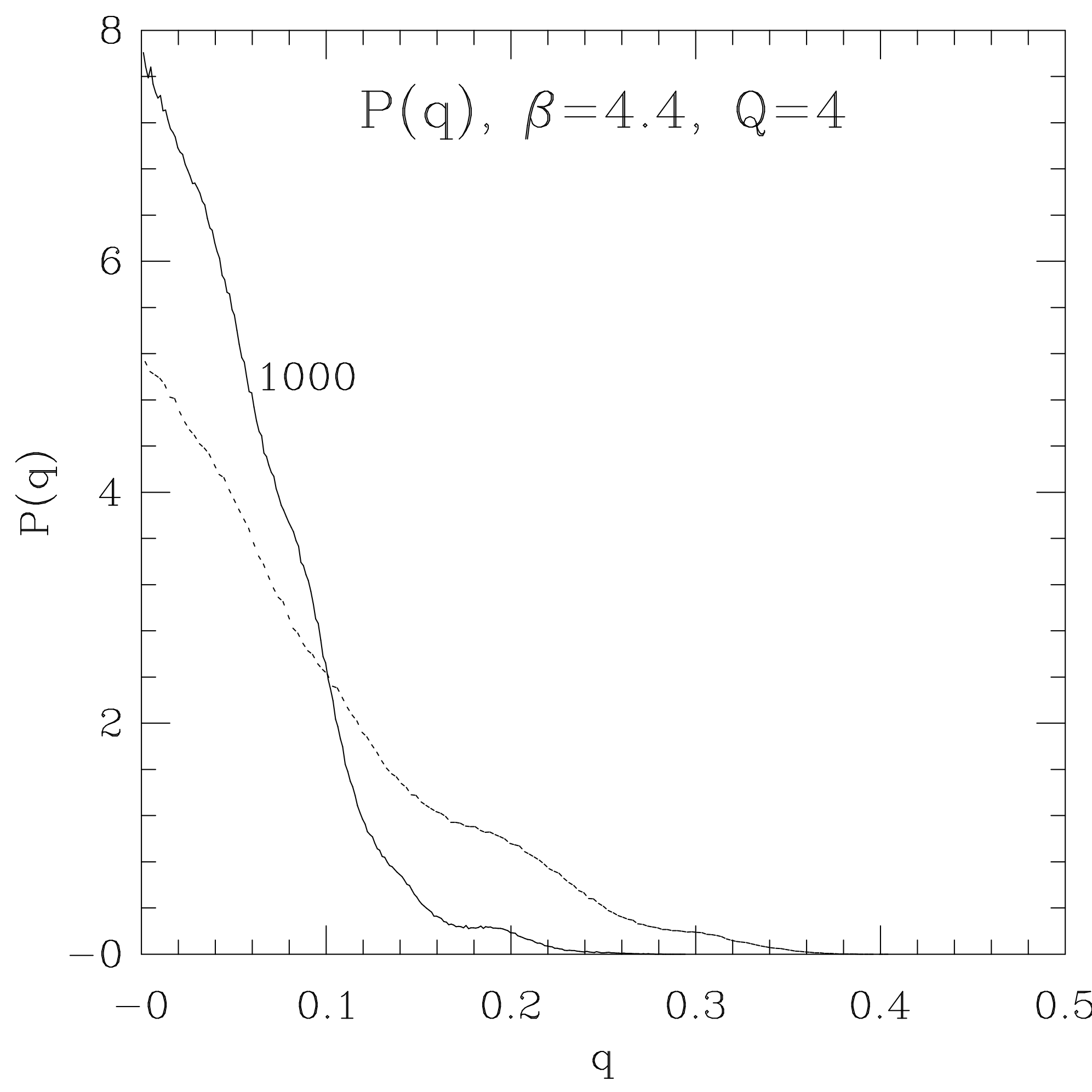




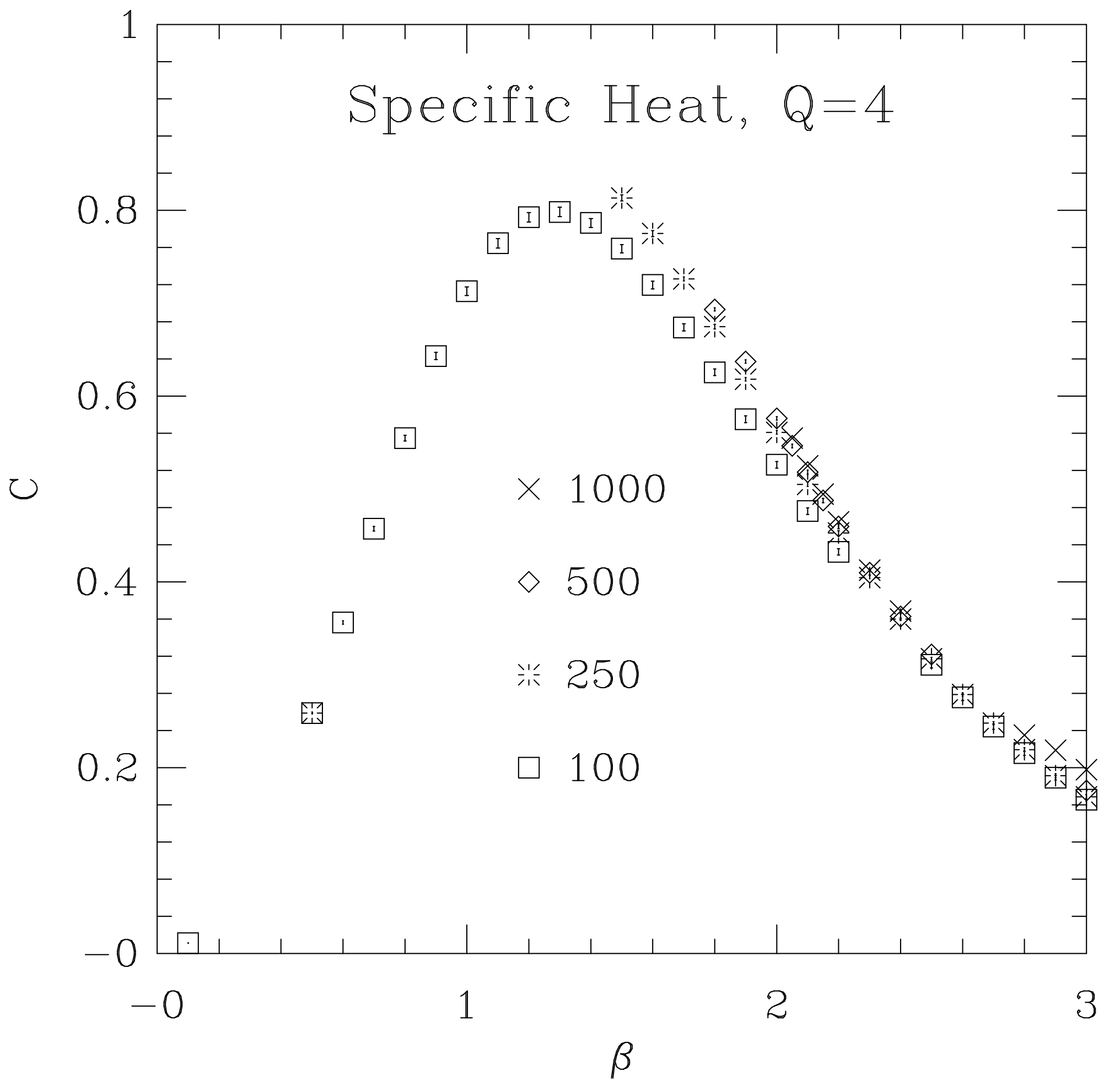

